\title{
Temporal changes in the vaginal microbiota in self-samples and its association with persistent HPV16 infection and CIN2+
}

\author{
Malin Berggrund ${ }^{1}$, Inger Gustavsson', Riina Aarnio², Julia Hedlund Lindberg ${ }^{1}$, Karin Sanner ${ }^{2}$, Ingrid Wikström², \\ Stefan Enroth ${ }^{1}$, Ignas Bunikis ${ }^{3}$, Matts Olovsson ${ }^{2}$ and Ulf Gyllensten ${ }^{1 *}$
}

\begin{abstract}
Background: The vaginal microbiota has been reported to be associated with HPV infection and cervical cancer. This study was performed to compare the vaginal microbiota at two timepoints in women performing self-sampling and had a persistent or transient HPV16 infection. The women were tested for 12 high-risk HPV (hrHPV) types but only women with single type (HPV16) were included to reduce confounding variables.
\end{abstract}

Methods: In total 96 women were included in this study. Of these, 26 were single positive for HPV16 in the baseline test and HPV negative in the follow-up test and 38 were single positive for HPV16 in both tests and diagnosed with CIN2+ in histology. In addition, 32 women that were negative for all $12 \mathrm{HPV}$ tested were included. The samples of vaginal fluid were analyzed with the lon $16 \mathrm{~S}^{\mathrm{TM}}$ Metagenomics Kit and Ion $16 \mathrm{~S}^{\mathrm{TM}}$ metagenomics module within the lon Reporter $^{\mathrm{TM}}$ software.

Results: K-means clustering resulted in two Lactobacillus-dominated groups, one with Lactobacillus sp. and the other specifically with Lactobacillus iners. The two remaining clusters were dominated by a mixed non-Lactobacillus microbiota. HPV negative women had lower prevalence (28\%) of the non-Lactobacill dominant cluster in the baseline test, as compared to women with HPV16 infection (42\%) ( $p$ value $=0.0173$ ). Transition between clusters were more frequent in women with persistent HPV16 infection (34\%) as compared in women who cleared the HPV16 infection (19\%) $(p$ value $=0.036$ )

Conclusions: The vaginal microbiota showed a higher rate of transitioning between bacterial profiles in women with persistent HPV16 infection as compared to women with transient infection. This indicate an instability in the microenvironment in women with persistent HPV infection and development of $\mathrm{CIN} 2+$.

Keywords: Vaginal microbiota, Self-sampling, HPV, CIN2+, Cervical cancer, FTA card

\section{Introduction}

Persistent infection of human papilloma virus (HPV) is the main cause for development of cervical cancer [1]. A set of HPV types have oncogenic properties and are

\footnotetext{
*Correspondence: ulf.gyllensten@igp.uu.se

1 Department of Immunology, Genetics, and Pathology, Biomedical

Center, Science for Life Laboratory (SciLifeLab) Uppsala, Uppsala University, Box 815, 75108 Uppsala, Sweden

Full list of author information is available at the end of the article
}

considered high-risk HPV [2], with HPV16 being the most prevalent type in most regions [3].

In addition to HPV, other risk factors for cervical cancer is recognized, such as smoking, high parity and use of oral contraceptives [4]. The association between HPV infection and the vaginal microbiota is not fully understood. Using $16 \mathrm{~S}$ sequencing, the vaginal microbiota profiles has previously been described as five community state types (CST). CST I, II, III and V are dominated by 
Lactobacillus crispatus, L. gasseri, L. iners and L. jensenii, respectively, while CST IV is dominated by non-Lactobacillus bacteria [5]. CST IV is further subgrouped into CST IV-a and IV-b, with IV-a having modest proportions of Lactobacillus spp. and low proportions of strictly anaerobic bacteria, while IV-b having higher proportions of Atopobium, as well as Prevotella, Sneathia and Gardnerella [6].

Several studies report Atopobium vaginae and Gardnerella vaginalis to be associated with cervical interepithelial neoplasia (CIN) [7-9]. However, other results show that decline of Gardnerella abundance with HPV positivity and increasing grade of CIN is associated [10]. Lower abundance of Lactobacillus $s p$. and higher frequency of CST-IV is associated with HPV infection and grade of CIN in several studies [11-13]. CST II (L. gasseri-dominated) is related to fast HPV clearance rate, and CST IV-B with slow HPV clearance rate, in comparison to CST I [14]. Also, one study reports relative abundance of $L$. Crispatus to be associated with lower HPV detection rate, but finds no association between HPV and relative abundance of other Lactobacillus species or with Lactobacillus as a group [15].

The vaginal microbiota in women is known to also depend on covariates such as the menstrual cycle $[6,16]$, pregnancy [17], use of hormonal contraceptives [18], sexual behavior and smoking [19]. In addition, the results can be affected by technical variations such as choice of variable regions of the $16 \mathrm{~S}$ gene [20] and sequencing platform [21].

The present study was based on analyses of seven variable domains of the 16S RNA gene in single samples from women with HPV negative tests and paired vaginal samples collected 4-6 months apart from women with a persistent HPV16 infection and women with transient infections. Only women infected by a single hrHPV type (HPV16) were included to reduce confounding variables in the analysis. These women were also tested for HPV18, $31,33,35,39,45,51,52,56,58$ and 59 with negative results. The aim was to study the association of the vaginal microbiota with the course of HPV16 infection.

\section{Methods}

\section{Study population and samples}

Women in this study were selected from a previous randomized intervention study conducted between 2013 and 2015, that included non-pregnant women between 30 and 49 years of age from Uppsala County, Sweden [22]. To be eligible for the randomized study, the woman had to be between 30 and 49 years at entry (date of invitation), having no previous hysterectomy, no current pregnancy and no clinical test results (Pap smear cytology,
HPV test or histology) relating to cervical cancer registered within 1 year before the date of invitation.

In brief, the randomized intervention study consisted of a total of 36,390 women out of which 17,997 were randomized to repeated HPV-testing with self-sampling. 7997 women performed the 1st test with 7443 negative and 554 positive tests. The aim of the previous study was to compare the detection rate of CIN2+ in histology in women performing repeated self-sampling of vaginal fluid for HPV testing, with the rate of CIN2+ detection in women following the regular screening program based on Pap smear cytology. Women who were HPV positive $(\mathrm{N}=554)$ in their first test were asked to repeat the selfsampling in 4-6 months. 501 of the 554 women followed the study protocol and performed a 2nd HPV-test which resulted in 355 positive tests. Women that were HPV negative in the first $(\mathrm{N}=7443)$ or second HPV test $(\mathrm{N}=146)$ were referred to the regular screening program. For the present study we selected 26 women who were single infected by HPV16 in the baseline test and subsequently HPV negative in their follow-up test, and 38 women who were single infected by HPV16 in both the baseline and the follow-up tests, and later diagnosed as CIN2+ based on histology during the follow-up period of 18 months from invitation date. Finally, 32 age-matched women who had a single self-sample with a negative HPV test were also included. Women that were infected by HPV16 in their baseline test and HPV negative in their follow-up test were considered to have transient infections, while women with two single HPV16 positive samples together with CIN2+ histology were considered to have a persistent infection. The study was approved by the Regional Ethics Committee in Uppsala (Dnr 2012/099).

\section{Sample collection and processing}

The vaginal self-sampling procedure has been described earlier [23]. Briefly, the women performed sample collection using the Rovers ${ }^{\circledR}$ Viba-brush (Rover Medical Devices B.V., Oss, The Netherlands) and applied the sample to the indicating FTA elute micro card ${ }^{\mathrm{TM}}$ (art. no WB129308, GE Healthcare, Longwood Dr, Cardiff CF14 7YT, UK). The instruction was to (1) insert the Rovers Viba-brush approximately $5-10 \mathrm{~cm}$ into the vagina and gently turn it one full circle, (2) remove the brush and apply the vaginal sample to the FTA elute micro card ${ }^{\text {TM }}$ by placing the brush in the middle of the application area and rolling it one full circle across that area and, (3) airdry the FTA card for a few minutes, fold the lid, put it in the enclosed envelope and send it to the HPV laboratory by regular mail. All samples were analyzed at the Department of Immunology, Genetics and Pathology, Uppsala University, Sweden. The FTA cards were processed using an automated laboratory system (easyPunch STARlet, 
Hamilton Robotics, Via Crusch 8 CH-7402 Bonaduz, GR, Switzerland), where a robot arm picks up each card, takes a photograph of the sampling area and then identifies which parts of the card that contains the highest concentration of cellular material, using a machine learning software. The robot then positions the card in a punching device and collects four circular pieces of $3 \mathrm{~mm}$ diameter in a single well in a 96-well microtiter plate. DNA was extracted from the card punches as previously described $[24,25]$.

\section{HPV DNA typing}

A clinically validated qPCR-based assay, HPVIR [26], was used for HPV DNA testing. The test detects and quantifies the following HPV types: $16,18,31,33,35,39,45$, $51,52,56,58$ and 59. The limit of detection (LOD) for HPV is $10 \mathrm{HPV}$ copies per PCR. This test also detects and quantifies a human single copy gene (housekeeping gene), HMBS (Homo sapiens hydroxymethylbilane synthase; GenBank accession no. M95623.1) as a control for the amount of human cellular material. The LOD for human genomic DNA is 10 copies of HMBS per PCR.

\section{Colposcopy and histology}

The colposcopic evaluation included an identification of squamocolumnar junction and transformation zone (TZ) with application of $5 \%$ acetic acid and iodine solution. Biopsies were obtained from all the identified abnormal areas, and a blind biopsy was taken in women with normal colposcopy. All gynecological examinations were performed at the Clinic of Obstetrics and Gynecology, Uppsala University Hospital. Clinical classifications were according to SNOMED (Systematized, Nomenclature of Medicine; College of American Pathologists, Skokie, IL, USA) and the highest histological grade found in each patient was used for interpretation of the results.

\section{Sample preparation and 16S rRNA gene Ion Torrent amplicon sequencing}

$16 \mathrm{~S}$ hypervariable regions were amplified using Ion $16 \mathrm{~S}^{\mathrm{TM}}$ Metagenomics Kit (Thermo Fisher Scientific) following recommendations in the Ion $16 S^{\mathrm{TM}}$ Metagenomics Kit User Guide revision C.0. According to the manufacturer's instructions, $3.0 \mu \mathrm{l}$ of the FTA-card eluate was used as input to the amplification. After purification with Agencourt $^{\circledR}$ AMPure ${ }^{\circledR}$ XP beads, amplicons were quantified using Bioanalyzer instrument (Agilent) and DNA-concentrations in the products ranged from 1.6 to $77.3 \mathrm{ng} / \mu \mathrm{l}$. With the exception of one sample where $50 \mathrm{ng}$ was used, $100 \mathrm{ng}$ of the input material was used for the AB Library Builder $^{\mathrm{TM}}$ System (Thermo Fisher Scientific), following the guide Ion Xpress ${ }^{\mathrm{TM}}$ Plus and Ion Plus Library Preparation for the AB Library Builder ${ }^{\mathrm{TM}}$ System revision 5.0.
Prepared libraries were amplified for addition 5 cycles as described in Ion $16 S^{\mathrm{TM}}$ Metagenomics Kit User Guide revision C.0. Final libraries were quantified using the Fragment analyzer (Agilent) and prepared in the Ion Chef System (Thermo Fisher Scientific) before sequenced on the Ion S5 ${ }^{\mathrm{TM}}$ XL System (Thermo Fisher Scientific) on five Ion $530^{\mathrm{TM}}$ Chips (Thermo Fisher Scientific).

The primary data analysis was carried out with The Ion reporter version 5.6. Briefly, quality analysis was run requiring primer detection in both ends, the minimum alignment coverage of $90 \%$. The analysis was performed with the built in QIIME pipeline with protocol Metagenomics $16 \mathrm{~S}$ w1.1. Reference library Curated Greengenes v13.5 was used and a minimum alignment of $97 \%$ was required for genus identification and 99\% for species identification. If no identification at $99 \%$ could be made on species level but was achieved at genus level, genus level annotations were used. Difference between the two top hits was allowed to be maximum $0.2 \%$ to confirm species identification.

\section{Statistical analysis}

Statistical calculations were performed and figures generated using $R$ version 3.4.3 [27]. Read number for each operational taxonomic unit (OTU) was normalized for total number of reads for each sample, e.g. reported in proportions of total number of reads. Non-Lactobacillus bacteria were grouped by taxonomic level genus if information was available, otherwise they were grouped on taxonomic level family. Bacterial genus or family that represented less than $1 \%$ of the total were grouped as "Others" for non-Lactobacillus bacteria, and as "Lactobacillus sp.2" for Lactobacillus bacteria. K-means clustering were performed with four clusters, using the "kmeans"function and the results visualized as a heatmap using the "pheatmap"-package [28]. Shannon index for alpha diversity was calculated with the "vegan"-package [29]. Sankey plots were visualized with the package "rCharts" [30]. Binomial testing was used for comparing cluster distribution between sample groups. $p$ values were adjusted for multiple testing using the Bonferroni correction and $q$ values were considered significant if the $q$ value $<0.05$. Binomial testing was used for comparison of cluster transition proportions.

\section{Results}

\section{Sample characteristics}

The median time between the two self-sampling occasions were 162 days and the mean absolute deviation (mad) 14.8 days for women with transient HPV16 infection and 155.5 days $(\operatorname{mad}=30.4)$ for women with persistent HPV16 infection. The number of days between the two sample collections was not significantly different 
between women with transient and persistent infection ( $p$ value $=0.35$ ). Also, the mean age was not significantly different between HPV negative women (mean $=36.7$, $\mathrm{SD}=5.2$ ), women with transient HPV16 infection (mean $=35.9, \mathrm{SD}=5.2)$ and women with persistent HPV16 infection and CIN2+ $($ mean $=37.5, \mathrm{SD}=6.0, p$ value $=0.31-0.64)($ Table 1$)$.

\section{$16 \mathrm{~S}$ rRNA gene sequencing and comparison between $16 \mathrm{~S}$ regions V2-V9 and V3/V4}

The Ion $16 \mathrm{~S}^{\mathrm{TM}}$ Metagenomics Kit targets seven variable regions (V2, V3, V4, V6, V7, V8 and V9). The number of reads per sample across these seven regions was between 125,202 and $1,148,323$, with a mean of $515,814 \pm 190,890$ reads. Based on the analysis of all seven variable regions, the analysis identified 723 operational taxonomic units (OTUs), corresponding to 433 species, 188 genera and 102 families. When the analysis was based solely on regions $\mathrm{V} 3$ and $\mathrm{V} 4$, which is a set of $16 \mathrm{~S}$ variable regions frequently used for analysis, we identified 547 OTUs, representing 324 species, 157 genera and 66 families (Additional file 1: Figure S1a-c). For regions V3 and $\mathrm{V} 4$ the number of reads per sample was between 68,211 and 574,180 , with a mean of $277,874 \pm 103,597$. Thus, including the seven regions resulted in the identification of additional OTUs at all three taxonomic levels. For instance, using the seven regions resulted in the identification of 34 Lactobacillus species, whereas using only regions V3 and V4 resulted in 26 Lactobacillus species. Since the analysis based on seven regions identified a higher number of OTUs at all three taxonomic levels and, in particular, a higher number of Lactobacillus

Table 1 Basic characteristics of samples from HPV negative women, women with transient HPV16 infection and women with persistent HPV16 infection and CIN2+

\begin{tabular}{|c|c|c|c|}
\hline & HPV negative & $\begin{array}{l}\text { Transient } \\
\text { HPV16 } \\
\text { infection }\end{array}$ & $\begin{array}{l}\text { Persistent } \\
\text { HPV16 } \\
\text { infection }\end{array}$ \\
\hline Number of samples & 32 & 26 & 38 \\
\hline Mean age $(\mathrm{SD})$ & $36.7(5.2)$ & $35.9(5.2)$ & $37.5(6.0)$ \\
\hline Sampling distance ${ }^{b}(S D)$ & NA & $184(79)$ & $165(33)$ \\
\hline \multicolumn{4}{|l|}{ Comparisons } \\
\hline \multicolumn{4}{|c|}{ HPV16 positive vs. HPV negative ( $p$ values) } \\
\hline $\mathrm{Age}^{\mathrm{c}}$ & & 0.525 & 0.64 \\
\hline \multicolumn{4}{|c|}{ Persistent vs. transient ( $p$ values) } \\
\hline $\mathrm{Age}^{\mathrm{c}}$ & & & 0.309 \\
\hline Sampling distance ${ }^{c}$ & & & 0.352 \\
\hline
\end{tabular}

\footnotetext{
a Age in years, given as: mean (standard deviation, SD)

b Distance between base-line sample and follow-up sample in days, given as: mean (standard deviation, SD)

c Two-sided Wilcoxon's test
}

species, we based the subsequent analysis on all seven $16 \mathrm{~S}$ regions. Using all seven region the number of OTUs per sample varied between 5 and 215, with a mean of 53 OTUs per sample.

\section{K-means clustering and cluster characteristics}

All OTUs with an abundance below $1 \%$ within a sample were analyzed as a group. OTUs belonging to Lactobacillus sp. and non-Lactobacillus sp. were grouped separately. K-means clustering, using all samples, with four clusters resulted in two Lactobacillus-dominated clusters. In the first cluster, samples had OTUs with unclassified Lactobacillus sp. as the most dominant species, with a mean proportion of $76.6 \%(\min =45.9 \%, \max =99.3 \%)$, and in the second cluster samples were dominated by OTUs specifically classified as Lactobacillus Iners, with a mean proportion of $86.4 \%(\min =48.2 \%, \max =99.4 \%)$. In cluster 3 and 4, samples were dominated by a mixed non-Lactobacillus microbiota. In the third cluster most samples were dominated by Gardnerella or Prevotella and some samples were dominated by e.g. Atopobium, Snethia or Streptococcus, with a mean proportion for the dominant bacteria of $39.4 \%(\min =15.2 \%, \max =92.9 \%)$. Finally, in the fourth cluster ten of eleven samples were dominated by Gardnerella and the last sample by Aerococcus, with a mean proportion of the dominant bacteria of $69.8 \%(\min =43.0 \%, \max =99.4 \%)$ (Fig. 1a).

\section{Vaginal microbiome clusters in HPV negative women and those with transient or persistent HPV16 infection} In the baseline test, HPV negative women had a lower prevalence $(28 \%)$ of the non-Lactobacilli dominant cluster (cluster 3 and 4), as compared to women with an HPV16 infection (42\%). This difference was nominally statistically significant ( $p$ value $=0.0173$ ), but the difference did not remain significant when corrected for multiple testing (Bonferroni normalized $q$ value $=0.0519$ ) (Table 2). In the second sample, women with transient infection had higher prevalence (42\%) of the non-Lactobacilli dominant clusters (cluster 3 and 4) than those with persistent infection (29\%), but the difference was not statistically significant $(p$ value $=0.10)($ Fig. $1 b$, Table 2$)$.

\section{Comparison of alpha diversity between infection groups}

Alpha diversity was calculated for each sample using the Shannon index, as an indicator of the variability of the vaginal microbiota. There was no significant difference between HPV negative women, women with transient HPV16 infection, and those with persistent HPV16 infection, neither for the baseline sample (all three groups of women) nor the second sample (the second two groups). All nominal $p$ values $>0.2$. 


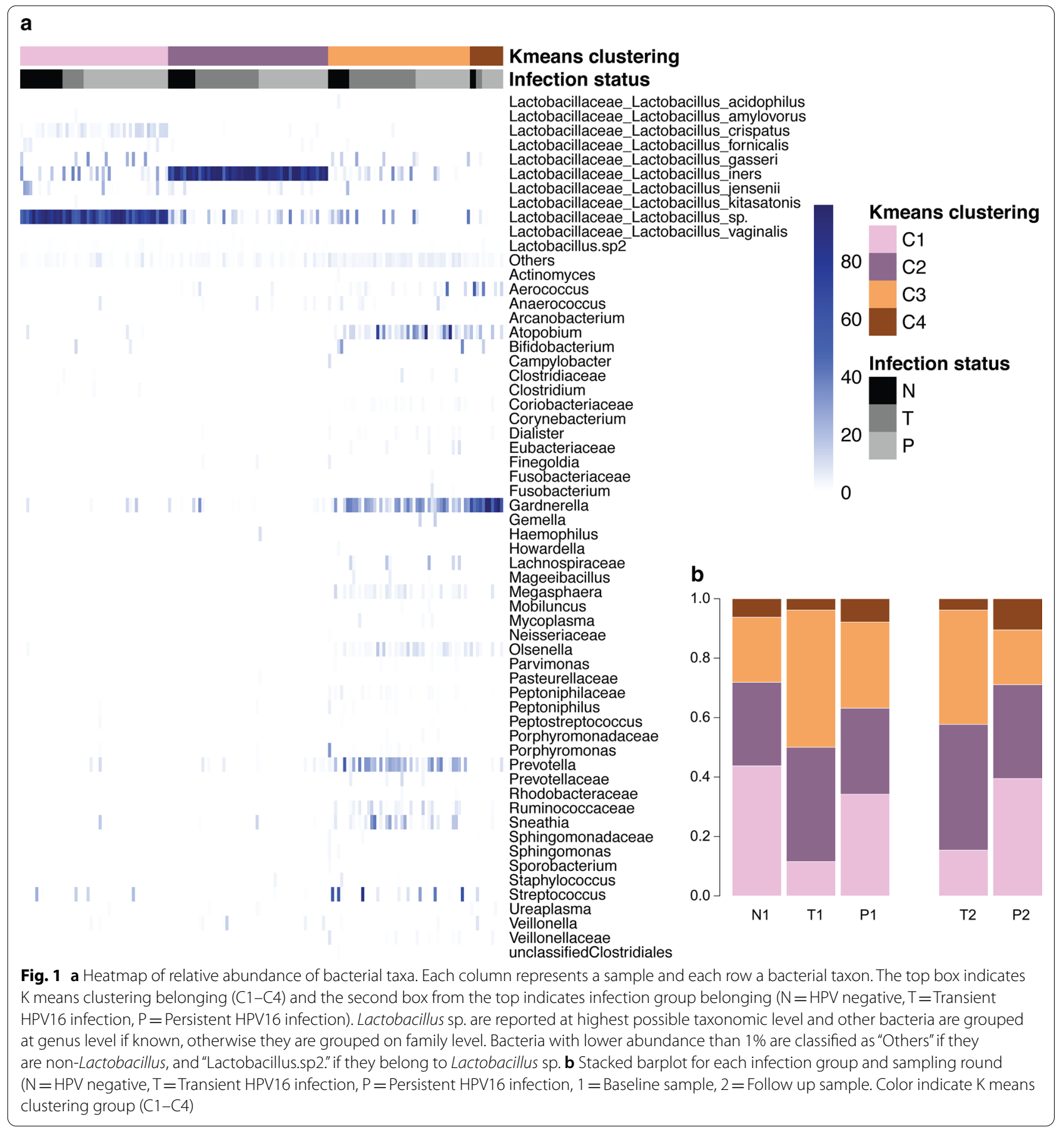

\section{Transition rate between microbial profiles in women} with transient or persistent HPV16 infection

We compared the K-means clusters in the baseline and second samples of individual women to study the transition between clusters as a function of clearance and persistence of HPV16 infection. Transition between clusters were significantly more frequent in women with persistent HPV16 infection (34\%) as compared to women who cleared the HPV16 infection in the timespan between the two sampling occasions (19\%) ( $p$ value $=0.036$, Fig. $2 a, b)$.

\section{Discussion}

A vaginal microbiota dominated by non-Lactobacillus species has been reported to be associated both with the risk of HPV infection and persistence of HPV infection [11-13] and is associated with up to three times higher 
Table 2 I. Distribution of HPV negative women, women with transient HPV16 infection and women with persistent HPV16 infection and CIN2+ in the four clusters. Proportion of total number of samples given in percent are reported within parenthesis. II. Statistical comparisons of the frequencies of non-Lactobacillus clusters (cluster 3 and 4 ) based on baseline samples of HPV16 negative women versus those with transient and persistent HPV16 infection, and for the follow up sample between women with transient HPV16 and persistent HPV16 infection

I. Distribution of samples per cluster

\begin{tabular}{|c|c|c|c|c|}
\hline & Cluster \#1 & Cluster \#2 & Cluster \#3 & Cluster \#4 \\
\hline HPV negative & $14(43.8)$ & $9(28.1)$ & $7(21.9)$ & $2(6.3)$ \\
\hline HPV16 transient, baseline sample & $3(11.5)$ & $10(38.5)$ & $12(46.2)$ & $1(3.8)$ \\
\hline HPV16 persistent, baseline sample & $13(34.1)$ & $11(28.9)$ & $11(28.9)$ & $3(7.9)$ \\
\hline HPV16 transient, follow up sample & $4(15.4)$ & $11(42.3)$ & $10(38.5)$ & $1(3.8)$ \\
\hline HPV16 persistent, follow up sample & $15(39.5)$ & $12(31.6)$ & $7(18.4)$ & $4(10.5)$ \\
\hline \multicolumn{5}{|l|}{ II. Statistical comparisons } \\
\hline & $p$ value & $q$ value & & \\
\hline \multicolumn{5}{|l|}{ a. Baseline sample } \\
\hline $\begin{array}{l}\text { HPV16 negative versus transient and persistent HPV16 } \\
\text { infection }^{\text {a }}\end{array}$ & 0.0173 & 0.0519 & & \\
\hline Transient HPV16 versus persistent HPV16 infection ${ }^{a}$ & 0.1433 & 0.4299 & & \\
\hline \multicolumn{5}{|l|}{ b. Follow up sample } \\
\hline Transient HPV16 versus persistent HPV16 infection ${ }^{a}$ & 0.423 & 1 & & \\
\hline
\end{tabular}

$p$ value for binomial testing

${ }^{a}$ Two-sided binomial testing

\section{a}

C1

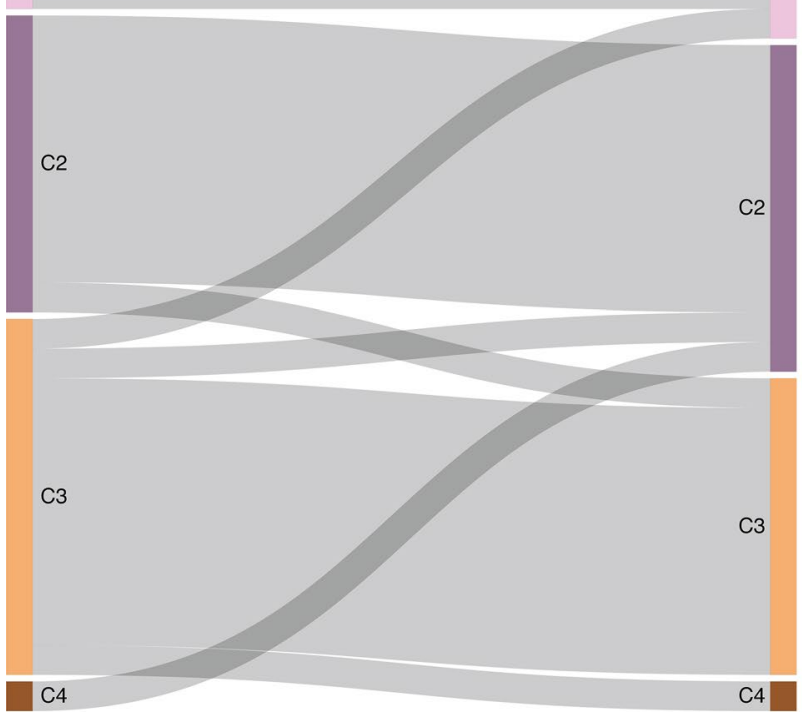

b

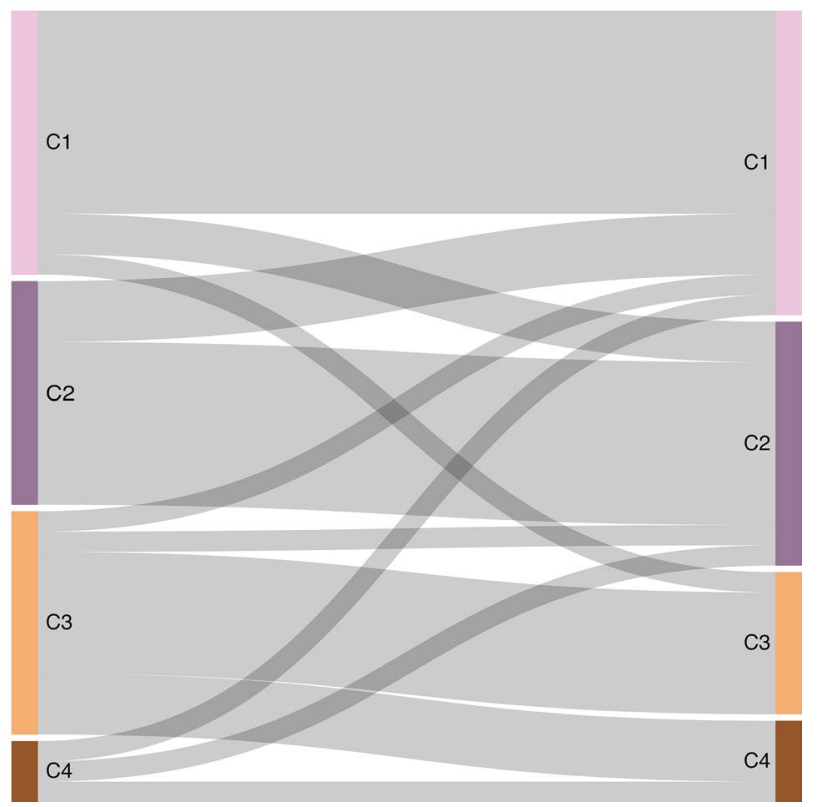

Fig. 2 Sankey plot for transition events between baseline and follow up sample for $\mathrm{K}$ means clusters. Left column represents baseline sample and right column follow up sample. Color and text indicate K means cluster. a Women with HPV16 transient infection. b Women with persistent HPV16 infection and $\mathrm{CIN}_{2+}$ 
odds for HPV infection and dysplasia [31]. In this study, we evaluated differences in vaginal microbiota in women with persistent single HPV16 infection, represented by women with two consecutive HPV16 positive tests and histologically diagnosed CIN2+ lesions, with women who cleared their HPV16 infection within 5-6 months, and a group of women who were HPV negative in the baseline test.

K-means clustering resulted in one cluster with Lactobacillus sp. dominance, a second cluster with Lactobacillus Iners dominance, a third cluster with a more diverse distribution of bacteria, and a fourth cluster also with a diverse distribution of bacteria but a higher abundance of Gardnerella. These groups are similar to previously described clusters $[5,6]$.

The results indicate that transient and persistent HPV16 infections, as compared to being HPV negative, is associated with a vaginal microbiota dominated by nonLactobacillus species ( $p$ value $=0.0173$ ). This is consistent with the results of several other studies [11-13]. However, the association did not remain significant after Bonferroni correction for multiple testing ( $q$ value $=0.0519$ ). We did not detect a difference in alpha diversity between women with or without HPV16 infection, nor did we find a difference between women who cleared their HPV16 infection and those with persistent HPV16 infections. This is consistent with one previous study [32], but other studies have reported the opposite [33, 34].

The vaginal microbiota profile did not differ significantly when comparing women who cleared their infection with those that had a persistent infection and CIN2+. However, there was a significantly higher rate of transitioning between microbiota profiles in women with persistent HPV16 infection, as compared to women who cleared their infection. This indicate an instability in the vaginal microenvironment in women with a persistent $\mathrm{HPV}$ infection and CIN2+.

The causative relationship between HPV infection and vaginal microbiota is poorly understood, but the inflammatory process in women with $\mathrm{CIN} 2+$ could have an effect on the vaginal microenvironment and the composition of the vaginal microbiota. The initial HPV infection in the basal layer has no productive life cycle before the differentiation of the infected cells, it contains no virus induced cell-death and causes limited inflammatory response [35]. The HPV virus also have immune evasion mechanism. For example, it has been shown that HPV infection halt downstream release of signals from viral RNA receptors, resulting in an inhibition of the inflammatory process [36] and downregulates IL-1 $\beta$, which is involved in the adaptive immune system [36, 37]. However, during a persistent HPV infection and development of CIN, the inflammatory process intensifies $[38,39]$, which could possibly influence the stability of the vaginal microenvironment and microbiota.

The strength of our study is that the women included were single-infected with HPV16, which reduces the complexity of HPV infection with different HPV types. We used HPV16 infected women that developed CIN2+ as a proxy for persistent infection. These women are likely to have harbored their HPV16 infections for some time, since they had developed CIN2+. There are some limitations in this study. The limited sample size reduces the statistical power to detect actual associations between the vaginal microbiota and the presence of HPV16. The HPV test include 12 high-risk HPV types, but the samples could possibly have other HPV infections that could affect the microbiota, which would go undetected with the test used in this study. Self-sampling could also potentially detect HPV-infections not exclusively present in the endocervix which would lead to an overestimation of detected HPV-infections. Here, this does not affect the negative or persistent group with CIN2+ histology diagnosis but may have influenced the positive HPV16 test for the first test in the transient group. A recent meta-review [40] does however conclude that PCR-based hrHPV tests on self-samples have similar sensitivity as clinician samples although a $2 \%$ lower specificity for CIN2+ could be observed.

We also lack information on whether the women participating in the study have been vaccinated for HPV or not. However, the age groups participating in the study have not been included in the organized Swedish HPVvaccination program which was initiated in 2012 for girls born in 1998-2000 [41] and vaccination rates among the women participating in the current study, all born before 1986, are assumed to be low. Finally, we lack information about co-variates that could affect the vaginal microbiota, such as smoking status, menstrual cycle time point, sexual behavior, use of hormonal contraceptives or copper intrauterine devices and ethnicity [6, 16-19]. Previous investigations have however shown small, or no significant effects of the menstrual cycle stage or of use of hormonal contraceptives on the vaginal microbiota [42, 43] although effects have been observed coupled to the use of copper intrauterine devices [43]. Sexual behavior has also been shown to affect the vaginal microbiota [44] and not being able to adjust for these factors may also have reduced the statistical power to detect differences between study groups.

We used the seven variable regions included in the Ion $16 \mathrm{~S}^{\mathrm{TM}}$ Metagenomics Kit. As noted, there is a difference in the number of OTUs identified at all taxonomic levels when the analysis is based on all seven variable regions as compared to the frequently employed regions V3 and V4. This can be attributed to the variability of the $16 \mathrm{~S}$ gene, 
the differences in primer design and sequencing technology makes direct comparisons between studies of the vaginal microbiota difficult. However, the use of seven regions resulted in a higher number of OTUs identified and generally had a good correspondence with the CSTs previously described for the vaginal microbiota.

In summary, to our knowledge this is the first report showing a significantly higher rate of transitioning between vaginal microbiota profiles in women with persistent HPV16 infection and CIN2+ as compared to women who cleared their infection.

\section{Conclusion}

Women with persistent HPV16 infection and CIN2+ have a higher rate ( $p$ value $=0.036$ ) of transitions between microbial profiles than women with transient HPV16 infection. This indicate a higher instability in the vaginal microenvironment in women with persistent HPV infection and development of CIN2+.

\section{Supplementary information}

Supplementary information accompanies this paper at https://doi. org/10.1186/s12985-020-01420-z.

Additional file 1. Figure S1: Venn diagram showing the overlap between the number of OTUs detected using seven variable regions (V2, V3, V4, V6, V7, V8 and V9) (blue) and the regions V3 and V4 only (yellow) based on the $16 \mathrm{~S}$ rRNA Ion Torrent amplicon sequencing kit. Figures represents the taxonomic level: A. Species, B. Genus, C. Family.

\section{Abbreviations}

CIN2+: Cervical intraepithelial neoplasia grade 2 or worse; CST: Community state types; HPV: Human papilloma virus; OTU: Operational taxonomic units; PCR: Polymerase chain reaction.

\section{Acknowledgements}

The authors would like to acknowledge support of the National Genomics Infrastructure (NGI_Uppsala Genome Center) for providing assistance with massive parallel sequencing and UPPMAX for the computational infrastructure. NGl/Uppsala Genome Center is supported by the Council for Research Infrastructures (RFI) at the Swedish Research Council (VR) and the Science for Life Laboratory, Sweden.

\section{Authors' contributions}

MB designed the study, designed and performed the statistical analyses, and wrote the paper. IB performed the bioinformatical analysis of the $16 \mathrm{~S}$ data. $I G$ and JHL performed and interpreted the HPV analyses. RA performed the colposcopies. KS, IW, SE, and MO interpreted the results. UG conceived of the study, contributed data and designed experiments. All authors contributed to the final version of the paper.

\section{Funding}

Open Access funding provided by Uppsala University. The study was supported by grants from the Swedish Cancer Foundation, the Swedish Research Council and the Foundation for Strategic Research (SSF) (to U.G.). The funding sources above together supported all aspects of the study including collection of data, analysis and interpretation, but none of the funding sources had any impact on the execution of the study, interpretation of the results and writing of the paper.

\section{Availability of data and materials}

The 165 sequencing data (sequencing reads or microbiome profile) will be made available from the corresponding author (to U.G.) upon request.
Ethics approval and consent toparticipate

The study was approved by the Regional Ethics Committee in Uppsala, Sweden (Dnr 2012/099). The women gave their informed consent when participating and sending in their self-collected samples.

\section{Consent to publication}

Not applicable.

\section{Competing interests}

The authors declare no financial or non-financial interests.

\section{Author details}

${ }^{1}$ Department of Immunology, Genetics, and Pathology, Biomedical Center, Science for Life Laboratory (SciLifeLab) Uppsala, Uppsala University, Box 815, 75108 Uppsala, Sweden. ${ }^{2}$ Department of Women's and Children's Health, Uppsala University, 75185 Uppsala, Sweden. ${ }^{3}$ Uppsala Genome Center, Science for Life Laboratory, Department of Immunology, Genetics, and Pathology, Uppsala University, BMC, Box 815, 75237 Uppsala, Sweden.

Received: 12 April 2020 Accepted: 28 September 2020

Published online: 07 October 2020

\section{References}

1. Walboomers JM, et al. Human papillomavirus is a necessary cause of invasive cervical cancer worldwide. J Pathol. 1999;189(1):12-9.

2. S. de Sanjose, M. Brotons, and M.A. Pavon, The natural history of human papillomavirus infection. Best Pract Res Clin Obstet Gynaecol, 2017. 47:2-13

3. de Martel C, et al. Worldwide burden of cancer attributable to HPV by site, country and HPV type. Int J Cancer. 2017;141(4):664-70.

4. Castellsague X, Bosch FX, Munoz N. Environmental co-factors in HPV carcinogenesis. Virus Res. 2002;89(2):191-9.

5. Ravel J, et al. Vaginal microbiome of reproductive-age women. Proc Natl Acad Sci U S A. 2011;108(Suppl 1):4680-7.

6. Gajer P, et al. Temporal dynamics of the human vaginal microbiota. Sci Transl Med. 2012:4(132):132ra52.

7. Godoy-Vitorino F, et al. Cervicovaginal fungi and bacteria associated with cervical intraepithelial neoplasia and high-risk human papillomavirus infections in a hispanic population. Front Microbiol. 2018;9:2533.

8. Curty $\mathrm{G}$, et al. Analysis of the cervical microbiome and potential biomarkers from postpartum HIV-positive women displaying cervical intraepithelial lesions. Sci Rep. 2017;7(1):17364.

9. Di Paola M, et al. Characterization of cervico-vaginal microbiota in women developing persistent high-risk human papillomavirus infection. Sci Rep. 2017;7(1):10200.

10. Audirac-Chalifour A, et al. Cervical microbiome and cytokine profile at various stages of cervical cancer: a pilot study. PLOS ONE. 2016;11(4):e0153274.

11. Shannon B, et al. Association of HPV infection and clearance with cervicovaginal immunology and the vaginal microbiota. Mucosal Immunol. 2017;10(5):1310-9.

12. Mitra A, et al. The vaginal microbiota, human papillomavirus infection and cervical intraepithelial neoplasia: what do we know and where are we going next? Microbiome. 2016;4(1):58

13. Lee JE, et al. Association of the vaginal microbiota with human papilIomavirus infection in a Korean twin cohort. PLoS ONE. 2013;8(5):e63514.

14. Brotman RM, et al. Interplay between the temporal dynamics of the vaginal microbiota and human papillomavirus detection. J Infect Dis. 2014;210(11):1723-33.

15. Reimers $L L$, et al. The cervicovaginal microbiota and its associations with human papillomavirus detection in HIV-infected and HIV-uninfected women. J Infect Dis. 2016;214(9):1361-9.

16. Srinivasan $\mathrm{S}$, et al. Temporal variability of human vaginal bacteria and relationship with bacterial vaginosis. PLoS ONE. 2010;5(4):e10197.

17. Romero R, et al. The composition and stability of the vaginal microbiota of normal pregnant women is different from that of non-pregnant women. Microbiome. 2014;2(1):4. 
18. Rifkin SB, et al. Hormonal contraception and risk of bacterial vaginosis diagnosis in an observational study of women attending STD clinics in Baltimore. MD Contracep. 2009;80(1):63-7.

19. Cherpes $T L$, et al. A delicate balance: risk factors for acquisition of bacterial vaginosis include sexual activity, absence of hydrogen peroxideproducing lactobacilli, black race, and positive herpes simplex virus type 2 serology. Sex Transm Dis. 2008;35(1):78-83.

20. Watts GS, et al. $16 \mathrm{~S}$ rRNA gene sequencing on a benchtop sequencer: accuracy for identification of clinically important bacteria. J Appl Microbiol. 2017;123(6):1584-96.

21. Salipante SJ, et al. Performance comparison of Illumina and ion torrent next-generation sequencing platforms for 16S rRNA-based bacterial community profiling. Appl Environ Microbiol. 2014;80(24):7583-91.

22. Gustavsson I, et al. Randomised study shows that repeated selfsampling and HPV test has more than two-fold higher detection rate of women with CIN2+ histology than Pap smear cytology. Br J Cancer. 2018;118(6):896-904.

23. Gustavsson I, et al. Type-specific detection of high-risk human papillomavirus (HPV) in self-sampled cervicovaginal cells applied to FTA elute cartridge. J Clin Virol. 2011;51(4):255-8.

24. Gustavsson I, et al. Use of FTA card for dry collection, transportation and storage of cervical cell specimen to detect high-risk HPV. J Clin Virol. 2009;46(2):112-6.

25. Gustavsson I, et al. Comparison between the hybrid capture 2 and the hpVIR real-time PCR for detection of human papillomavirus in women with ASCUS or low grade dysplasia. J Clin Virol. 2009;45(2):85-9.

26. Gustavsson I, et al. Clinical validation of the HPVIR high-risk HPV test on cervical samples according to the international guidelines for human papillomavirus DNA test requirements for cervical cancer screening. Virol J. 2019;16(1):107.

27. R Core Team. R: A Language and Environment for Statistical Computing. Vienna: R Foundation for Statistical Computing; 2014.

28. Kolde R. Pheatmap: pretty heatmaps. R Package Version. 2012;61:1-7.

29. Jari Oksanen RK, Legendre P, O'Hara B, Henry M, Stevens H. Maintainer Jari Oksanen, MASS Suggests. The vegan package Community Ecology Package. 2007;10:631-7.

30. Vaidyanathan R. rCharts. R Package, 2016.

31. Norenhag J, et al. The vaginal microbiota, human papillomavirus and cervical dysplasia: a systematic review and network meta-analysis. BJOG. 2019;127(2):171-80.

32. Onywera $\mathrm{H}$, et al. The cervical microbiota in reproductive-age South African women with and without human papillomavirus infection. Papillomavirus Res. 2019;7:154-63.
33. Di Pietro M, et al. HPV/Chlamydia trachomatis co-infection: metagenomic analysis of cervical microbiota in asymptomatic women. New Microbiol. 2018;41(1):34-41.

34. Arokiyaraj $\mathrm{S}$, et al. Association of cervical microbial community with persistence, clearance and negativity of human papillomavirus in Korean women: a longitudinal study. Sci Rep. 2018;8(1):15479.

35. Moody CA, Laimins LA. Human papillomavirus oncoproteins: pathways to transformation. Nat Rev Cancer. 2010;10(8):550-60.

36. Karim $\mathrm{R}$, et al. Human papillomavirus deregulates the response of a cellular network comprising of chemotactic and proinflammatory genes. PLoS ONE. 2011;6(3):e17848.

37. Niebler $\mathrm{M}$, et al. Post-translational control of IL-1 beta via the human papillomavirus type 16 E6 oncoprotein: a novel mechanism of innate immune escape mediated by the E3-ubiquitin ligase E6-AP and p53. PLoS Pathog. 2013;9(8):e1003536.

38. Hammes LS, et al. Macrophages, inflammation and risk of cervical intraepithelial neoplasia (CIN) progression-clinicopathological correlation. Gynecol Oncol. 2007;105(1):157-65.

39. Mhatre $M$, et al. Cervical intraepithelial neoplasia is associated with genital tract mucosal inflammation. Sex Transm Dis. 2012;39(8):591-7.

40. Arbyn $M$, et al. Detecting cervical precancer and reaching underscreened women by using HPV testing on self samples: updated meta-analyses. BMJ. 2018;363:k4823.

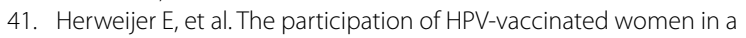
National Cervical Screening Program: Population-Based Cohort Study. PLOS ONE. 2015;10(7):e0134185.

42. Chaban B, et al. Characterization of the vaginal microbiota of healthy Canadian women through the menstrual cycle. Microbiome. 2014;2:23.

43. Achilles SL, et al. Impact of contraceptive initiation on vaginal microbiota. Am J Obstet Gynecol. 2018;218(6):622e1-622e10.

44. Wessels JM, et al. Association of high-risk sexual behaviour with diversity of the vaginal microbiota and abundance of Lactobacillus. PLOS ONE. 2017;12(11):e0187612.

\section{Publisher's Note}

Springer Nature remains neutral with regard to jurisdictional claims in published maps and institutional affiliations.
Ready to submit your research? Choose BMC and benefit from:

- fast, convenient online submission

- thorough peer review by experienced researchers in your field

- rapid publication on acceptance

- support for research data, including large and complex data types

- gold Open Access which fosters wider collaboration and increased citations

- maximum visibility for your research: over $100 \mathrm{M}$ website views per year

At BMC, research is always in progress.

Learn more biomedcentral.com/submissions 